\title{
Use of X-ray Charge Densities in the Calculation of Intermolecular Interactions and Lattice Energies: Application to Glycylglycine, DL-Histidine, and DL-Proline and Comparison with Theory
}

\author{
Yuriy A. Abramov,* Anatoliy Volkov, Guang Wu, and Philip Coppens* \\ Department of Chemistry, State University of New York at Buffalo, Buffalo, New York, 1460-3000
}

Received: December 9, 1999

\begin{abstract}
Experimental X-ray charge densities from low-temperature data are used in the evaluation of the intermolecular interactions and lattice energies of crystals of glycylglycine, DL-histidine, and DL-proline. The X-ray analysis leads to a set of atom-centered distributed multipoles, from which electrostatic interactions are calculated. Nonempirical exp-6 atom-atom potentials are used to calculate the smaller contributions of van der Waals interactions. For comparison, parallel theoretical calculations are performed on the molecular dimers (B3LYP) and the periodic crystals (Periodic Hartree-Fock, PHF). The dimer interactions show good agreement with experimental values, except for the strongest interactions in the glycylglycine crystal. The experimental charge density results correlate well with those based on the PHF calculations, but quantitative agreement for the interaction energies is only obtained after application of a scaling factor of $\sim 0.76$ to the PHF values. The discrepancy is attributed to the well-known overestimate of molecular polarity in the HF method, resulting from neglect of electron correlation. The agreement between lattice energies derived from the experimental charge density and theoretical values from the PHF calculations is within $10 \mathrm{~kJ} / \mathrm{mol}$ for the crystals examined in this study. The study provides the basis for use of experimental electrostatic moments in molecular modeling calculations of more complex systems.
\end{abstract}

\section{Introduction}

In the past few years, considerable progress has been made in the measurement of charge densities in crystals. ${ }^{1}$ As the development of area detectors has speeded up data collection, the time required for the collection of a typical charge density data set has been reduced to a few days or less., ${ }^{2,3}$ A new software package for aspherical atom multipole refinement of the experimental data and analysis of the results has gained widespread acceptance. ${ }^{4}$ As a result, experimental charge density analysis has become a tool in the study of problems of chemical and physical interest.

The experimental charge density intrinsically includes correlation effects such as dispersion, which are often unavailable from ab initio calculations of periodic systems. One of its applications is the evaluation of electrostatic properties of molecules in crystals, including the energy of intermolecular electrostatic interactions. In the experimental charge density approach (ECDA) to intermolecular interactions, ${ }^{5}$ the anisotropic electrostatic interactions are derived from the atom-centered multipoles $\left(E_{\mathrm{es}}^{\mathrm{mul}}\right)$ and combined with isotropic atom-atom exp-6 potentials for the van der Waals interactions $\left(U_{\mathrm{vdW}}\right)$ :

$$
\begin{gathered}
E_{\mathrm{int}}=E_{\mathrm{es}}^{\mathrm{mul}}+U_{\mathrm{vdW}} \\
U_{\mathrm{vdW}}=\sum_{i j} A_{i j} \exp \left(-B^{*} R_{i j}\right)-C / R_{i j}{ }^{6}
\end{gathered}
$$

The first term of the exp-6 potential (2) describes the shortrange repulsion energy $\left(E_{\text {rep }}\right)$ between monomer charge distributions, while the second term represents the dispersion energy $\left(E_{\mathrm{dis}}\right)$. The atom-atom potentials of Spackman, employed here, are based on the electron gas model within the Kim and Gordon approximation $^{6}$ and are combined with approximate dispersion energy terms. ${ }^{7}$ As the Spackman potentials do not include exchange-repulsion terms for strong hydrogen bonds, we have included the exp- 6 potential of Price et al. ${ }^{8}$ for these interactions.

The atom-centered electrostatic moments from the X-ray analysis $^{1}$ are used with Buckingham's multipole expansion ${ }^{9}$ to evaluate the electrostatic interaction energy, $E_{\mathrm{es}}^{\mathrm{mul}}$. An intrinsic limitation of the point multipole expansion is that it is rigorous only for nonoverlapping charge distributions. The neglected electrostatic effect of the overlap of the charge densities, the penetration energy $E_{\text {pen, }}$ is always negative, because of the deshielding of the nuclei. Spackman introduced additional corrections to the $E_{\mathrm{es}}{ }^{\mathrm{mul}}$ term ${ }^{5}$ to account approximately for the overlap of charge densities. ${ }^{10}$ However, there is no clear understanding how closely such corrections approximate the actual penetration energy.

The presentation of the total interaction energy $\left(E_{\text {int }}\right)$ by eq 1 is based a perturbation theory expansion for monomermonomer interactions: ${ }^{11,12}$

$$
E_{\mathrm{int}} \approx E_{\mathrm{es}}+E_{\mathrm{rep}}+E_{\mathrm{pol}}+E_{\mathrm{ct}}+E_{\mathrm{dis}}+\ldots
$$

Here $E_{\mathrm{es}}$ is the electrostatic interaction energy between two unperturbed monomers; $E_{\mathrm{pol}}$ is the attractive polarization energy due to the response of one monomer to the electric field of the second monomer; $E_{\mathrm{ct}}$ is an attractive energy contribution due to charge transfer, which occurs in the case of two differing molecules.

In empirical force field models, the attractive $E_{\mathrm{pol}}, E_{\mathrm{ct}}$, and $E_{\text {pen }}$ terms tend to be absorbed in the empirical charges and atom-atom potentials. ${ }^{11}$ As the experimental charge density already includes the perturbation by the many-body interactions in the crystal, the first two of these, $E_{\mathrm{pol}}$ and $E_{\mathrm{ct}}$, are included in the evaluation of the electrostatic interactions. 

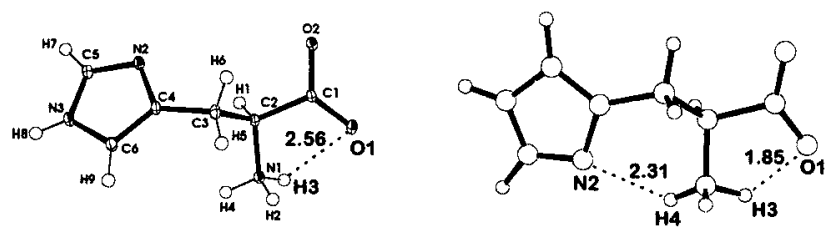

(a)
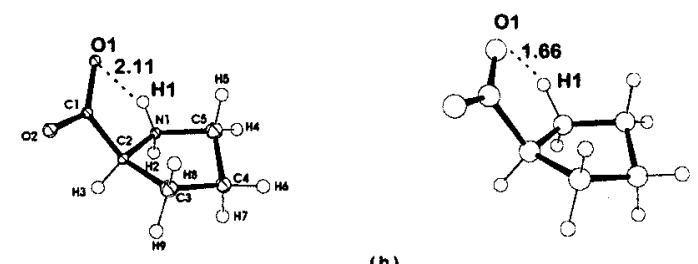

(b)

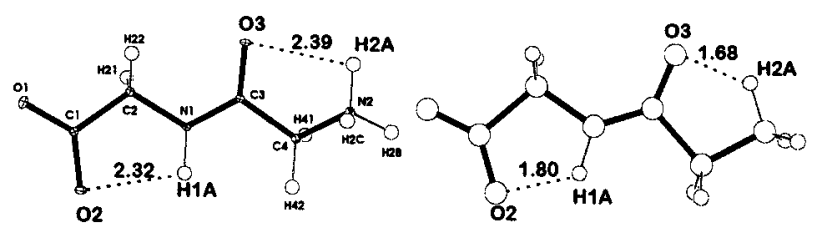

(c)

Figure 1. Crystal (left) and optimized (right) molecular structures of (a) histidine, (b) proline, and (c) glycylglycine. Optimization was performed at the HF/6-311G** level of theory. Intramolecular hydrogen bonds in the optimized molecular geometries are shown by dashed lines with the bond distances indicated in angstroms. For comparison, the corresponding interatomic distances are shown for the crystal geometries also.

Previously reported results on the ECDA approach to the intermolecular interaction energies in simple ${ }^{5,13}$ and more complex ${ }^{14}$ molecular crystals are encouraging. In the present ECDA study, recent improvements in experimental and calculational procedures are applied to the molecular crystals of DLhistidine, DL-proline, and glycylglycine. Special attention is paid to the basis set overlap error ${ }^{15}$ and to adequate treatment of the asphericity of the hydrogen atoms in the multipole refinement. The ECDA dimer interaction energies are compared with results from supermolecule calculations and calculations on periodic crystals. A similar comparison is made between the lattice energies from the experimental charge density and those from theoretical Hartree-Fock calculations on the periodic system.

\section{Experimental Section}

Crystal and Molecular Structures. High-quality X-ray diffraction experiments on the DL-histidine and DL-proline crystals were reported previously. ${ }^{14,2}$ The X-ray diffraction study of the glycylglycine will be described elsewhere. ${ }^{16}$ All experiments were performed at low temperatures $(110,20$, and $20 \mathrm{~K}$, respectively) using CCD-area detectors and synchrotron radiation in the last two cases. All crystals have centrosymmetric unit cells (the space groups are $P 2_{1} / c, P b c a$, and $P 2_{1} / c$, respectively), resulting in the presence of both $\mathrm{D}$ and $\mathrm{L}$ conformations of the histidine and proline molecules. There is one water molecule in the asymmetric unit of DL-proline. The molecular structures of histidine (His), proline (Pro), and glycylglycine (GlyGly) in the crystal are presented in Figure 1 (left).

The intermolecular bond paths in the crystals were identified by topological analysis ${ }^{17}$ of the experimental charge densities. Most of these contacts correspond to conventional hydrogen bonds. The geometry of the molecular pairs displaying intermolecular hydrogen bonding in the DL-proline and glycylglycine crystals are shown in Figure 2. The molecular pairs sharing intermolecular surfaces in the DL-histidine crystal have been reported previously. ${ }^{14}$

The Charge Density Multipole Model. The HansenCoppens multipole formalism, ${ }^{1}$ as implemented in the XD software package, ${ }^{4}$ was used for both observed and theoretical structure factor fitting. It describes the crystal electron density as a superposition of aspherical pseudoatoms each modeled by a multipole expansion:

$$
\rho_{k}(\mathbf{r})=\mathrm{P}_{\mathrm{c}} \rho_{\mathrm{c}}(r)+P_{\mathrm{v}} \kappa^{3} r_{\mathrm{v}}(\kappa r)+\sum_{l=1}^{4} \kappa^{\prime 3} R_{\mathrm{l}}\left(\kappa^{\prime} r\right) \sum_{m=-1}^{l} P_{1 \mathrm{~m}} d_{1 \mathrm{~m}}(\mathbf{r} / r)
$$

Here $\rho_{\mathrm{c}}$ and $\rho_{\mathrm{v}}$ are spherically averaged free-atom HartreeFock core and valence densities normalized to one electron; $d_{l m}$ are real spherical harmonic angular functions; $R_{l}$ are normalized Slater-type radial functions; and $\kappa$ and $\kappa^{\prime}$ are dimensionless expansion-contraction parameters, which can be refined in the fitting procedure along with the populations $P_{\mathrm{v}}$ and $P_{l m}$. The charge density asphericity was described at the octupole $(l=3)$ level for all non-hydrogen atoms and at the dipole and quadrupole levels ( $l=1$ or 2$)$ for hydrogen atoms not involved and involved in strong $\mathrm{H}$-bonds, respectively. To decrease the number of variables in the refinement, chemical and local symmetry constraints were applied. For example, atoms in the carboxylate, nitrite, phenyl, and imidazol groups were constrained to have mirror symmetry. Charge densities of all hydrogens were considered to have cylindrical symmetry along the corresponding hydrogen-heavy atom bond. A molecular electroneutrality constraint was applied in all refinements. Each component of the proline $-\mathrm{H}_{2} \mathrm{O}$ complex was considered to be neutral, in accordance with theoretical PHF calculations of the complex, which predict a negligible charge transfer $(-0.001 \mathrm{e})$.

The Intermolecular Interaction Model. The ECDA model used in the present study is a slightly modified version of that introduced by Spackman. ${ }^{5}$ In the case of polar molecules such as $\alpha$-amino acids, the electrostatic term $E_{\mathrm{es}}$ is expected to dominate the intermolecular interaction energy. ${ }^{1,18}$ This term was evaluated via the atom-centered multipole expansion up to octupole-octupole $\left(\sim R^{-7}\right)$ term in the Cartesian tensor formulation. ${ }^{1,9}$ The convergence of the multipole series was tested by examination of the contributions of terms with increasing order of $1 / R$. In the present study, the maximum contribution of the octupole-octupole terms is $1.4 \mathrm{~kJ} / \mathrm{mol}$.

The van der Waals interaction term, $E_{\mathrm{vdW}}$, was evaluated with the exp-6 atom-atom potentials (2) as parametrized by Spackman, ${ }^{7}$ complemented by the atom-atom potential for polarized hydrogen atoms $\left(\mathrm{H}_{\mathrm{p}}\right)$ participating in strong hydrogen bonds $\left(A_{\mathrm{H}_{\mathrm{p}} \mathrm{H}_{\mathrm{p}}}=7017.3 \mathrm{~kJ} / \mathrm{mol}, C_{\mathrm{H}_{\mathrm{p}} \mathrm{H}_{\mathrm{p}}}=16.4 \mathrm{~kJ} \AA^{6} / \mathrm{mol}, B_{\mathrm{H}_{\mathrm{p}} \mathrm{H}_{\mathrm{p}}}=4.66\right.$ $\left.\AA^{-1}\right) .8$

Theoretical Calculations. Periodic HF structure calculations were performed with the CRYSTAL95 software package. ${ }^{19}$ Two types of atomic basis sets were used. The first, 6-21G**, ${ }^{20,21}$ was successfully tested previously on the urea ${ }^{22}$ crystal. The second basis set, 6-31G**,21 has been used previously for periodic $\mathrm{HF}$ calculations of crystalline $\mathrm{HCN},{ }^{23}$ ice VIII, ${ }^{24}$ urea, ${ }^{25}$ and formamide. ${ }^{26}$ As a result of the computational limitations, the second set could not be applied to the DL-proline crystal (there are 160 atoms in the unit cell of DL-proline in comparison with 80 and 68 atoms in the unit cells of respectively the DLhistidine and glycylglycine). To avoid severe numerical instability in calculations with the $6-31 \mathrm{G}^{* *}$ set, the diffuse outermost Gaussian function of the hydrogen and carbon atoms were scaled 

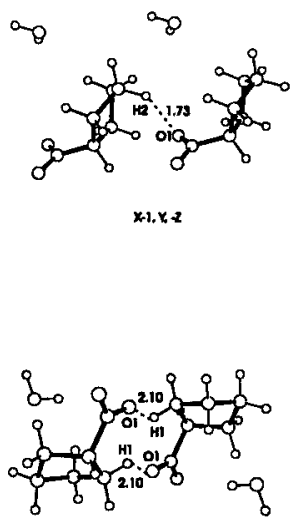

$x+x-z$

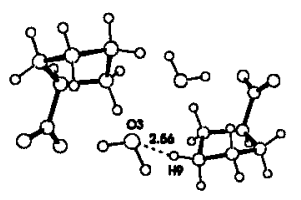

$* 18+7,122$

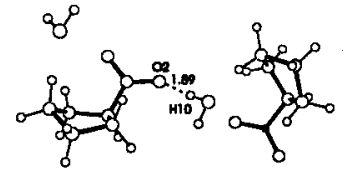

$12 \times \times 122$

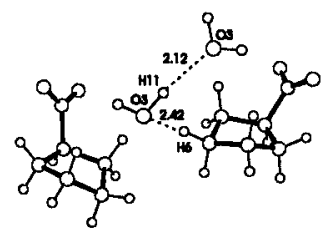

@

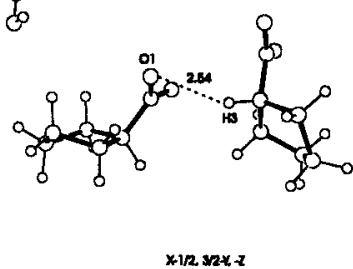

$x+1 / 2,2 x-2$
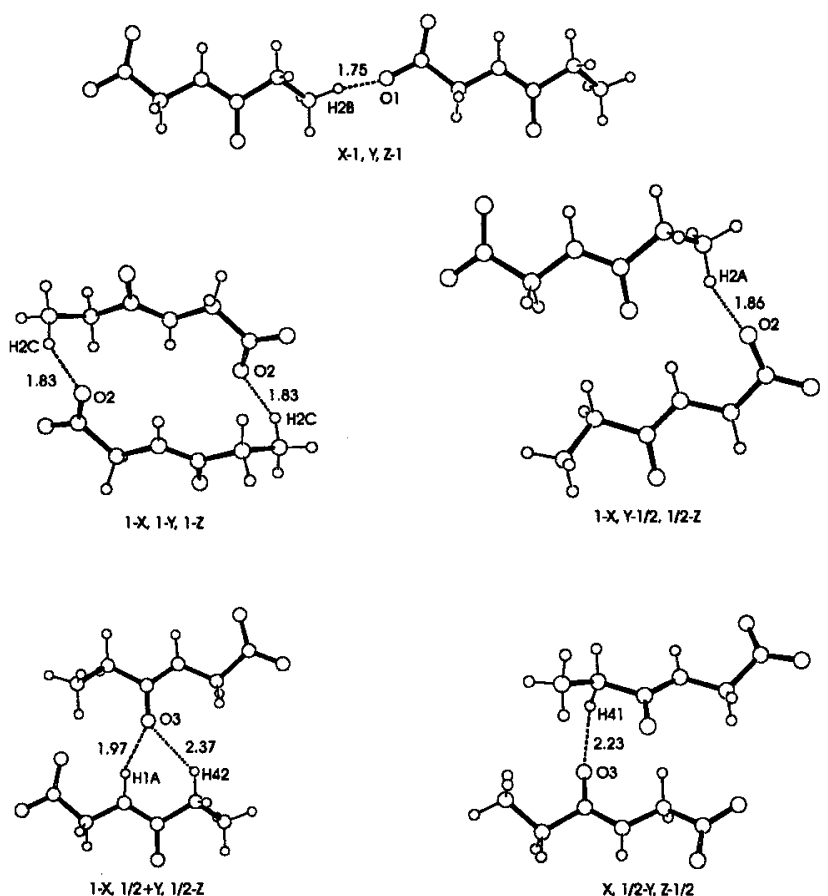

$1-X, 1 / 2+Y, 1 / 2-2$

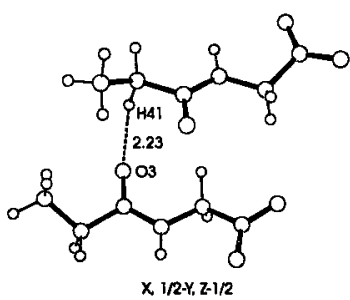

(b)

Figure 2. Geometry of the molecular pairs displaying hydrogen bonding in the DL-proline (a) and glycylglycine (b) crystals.

(the exponents were changed from $0.16127780(\mathrm{H})$ and 0.1687144 (C) to $0.2 \mathrm{bohr}^{-1}$ for the both atoms). ${ }^{27}$ The shrinking factor for the Monkhorst net ${ }^{28}$ was set equal to 8, giving 170, 125, and $170 k$ points in the reciprocal space of the DL-histidine, DL-proline, and glycylglycine crystals, respectively. The Gilat shrinking factor ${ }^{29}$ was set to 16 , and eight plane waves were used for the expansion of the eigenvalues $e_{k}$.

The lattice energy, $E_{\mathrm{L}}$, was evaluated from the following contributions:

$$
E_{\mathrm{L}}=E 1+E 2
$$

with

$$
\begin{gathered}
E 1=E_{\mathrm{mol}}(\text { crystal })-E_{\text {mol }}(\text { gas, crystal geometry }) \\
E 2=-\left(E_{\text {dissociation }}+E_{\text {relaxation }}\right) \\
E_{\text {relaxation }}=E_{\text {mol }}(\text { gas, optimized geometry })- \\
E_{\text {mol }}(\text { gas, crystal geometry })
\end{gathered}
$$

Here $E_{\text {mol }}$ (crystal) is the energy of the His, Pro $\cdot \mathrm{H}_{2} \mathrm{O}$, or GlyGly entities in the crystal. $E_{\text {mol }}$ (gas, crystal geometry) is the energy of the isolated entities with the crystal geometry. Both these energies were evaluated with CRYSTAL95. The basis set superposition error (BSSE) in E1 was corrected by adopting the counterpoise $(\mathrm{CP})$ method $^{30}$ (basis sets of all ghost atoms at a distance less than $3.5 \AA$ from the molecule were added in the $E_{\text {mol }}$ (gas, crystal geometry) calculations). $E_{\text {dissociation }}$ is energy of dissociation of the Pro $\cdot \mathrm{H}_{2} \mathrm{O}$ dimer into the isolated Pro and $\mathrm{H}_{2} \mathrm{O}$ molecules in the crystal geometry. $E_{\text {relaxation, }}$ the relaxation energy, is the difference between the energy of the isolated molecule and the molecule with the crystal geometry. All energy terms in E2 were calculated by the GAUSSIAN94 program package $^{31}$ at the HF/6-311G** level. The CP correction was applied in the $E_{\text {dissociation }}$ calculation. To allow comparison with the ECDA results, no crystal geometry optimizations were performed. Zero-point energy contributions to the lattice energy
TABLE 1: Comparison of the Experimental and Theoretical Dipole Moments $(D)$ of the Glycylglycine Molecule

\begin{tabular}{ccc}
\hline $\begin{array}{c}\text { experiment } \\
\text { (crystal) }\end{array}$ & $\begin{array}{c}\mathrm{HF} / 6-311 \mathrm{G}^{* *} \\
\text { (molecule) }\end{array}$ & $\begin{array}{c}\text { B3LYP/6-311G** } \\
\text { (molecule) }\end{array}$ \\
\hline $27.8(17)$ & 25.44 & 23.24
\end{tabular}

were ignored. In all cases, sets of static structure factors containing the same unique reflections as observed in the experiments were derived from the PHF theoretical electron density.

The theoretical calculations of the dimer interaction energies were performed with the GAUSSIAN94 program package at the density functional B3LYP/6-311G** level of theory. The experimental molecular geometry was used in these calculations.

\section{Refinement Strategy}

Basis Set Overlap Problem. Molecules in the crystals under consideration display significant charge density (basis set) overlap because of relatively strong intermolecular hydrogen bonding. We have shown that the lack of physical constraints in the purely mathematical multipole refinement model can lead to errors in the evaluation of the static molecular properties due to intermolecular overlap of the basis sets. ${ }^{15}$ This basis set overlap error is greatly reduced by use of the kappa restricted multipole model (KRMM), in which a fixed standard set of $\kappa^{\prime}$ values (note that $\kappa^{\prime}$ determines the radial extent of the deformation function but not that of the spherical valence shells), based on multipole refinement of several sets of static PHF structure factors, is used. ${ }^{15}$ The KRMM refinement produces a quite reasonable enhancement of the GlyGly molecular dipole moment in the crystal (Table 1). The molecular dipole moments of the DL-histidine and DL-proline have been discussed previously. ${ }^{15}$

Treatment of the Hydrogen Atoms. Since hydrogen bonding introduces one of the main stabilizing contributions to intermolecular interactions, the treatment of hydrogen atoms in the multipole model deserves special attention. In the absence of 


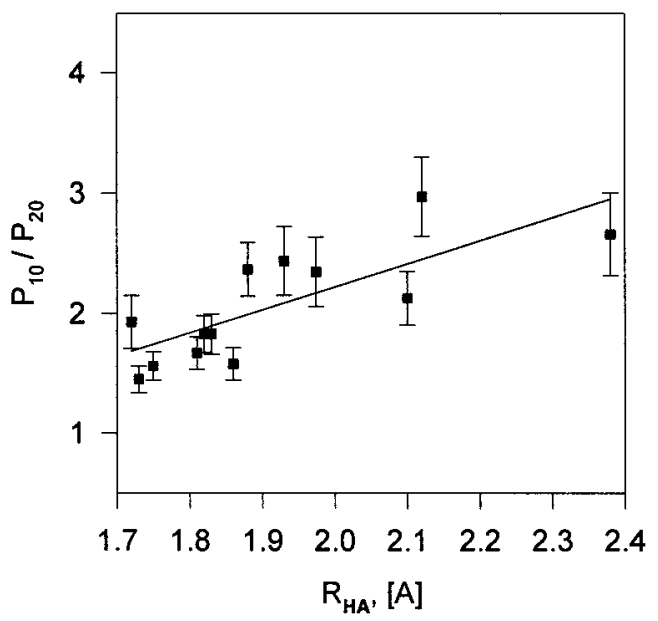

Figure 3. Dependence of the $\mathrm{P}_{10} / \mathrm{P}_{20}$ ratio of the hydrogen atom on the hydrogen to acceptor distance $R_{\mathrm{HA}}[\AA]$ according to the refinement of the theoretical structure factors for the three crystals discussed. The linear fit $(R=0.77)$ is given by $P_{10} / P_{20}=-1.62(92)+1.92(48) R_{\mathrm{HA}}$.

neutron data at the same temperature, the following strategy was developed.

(1) The hydrogen density functions were centered at the proton positions, obtained by extending the $\mathrm{X}-\mathrm{H}$ distances to standard neutron diffraction values $\left(\mathrm{C}-\mathrm{H}, 1.087 \AA\right.$; $\mathrm{N}\left(\mathrm{NH}_{3}\right)-$ H, $1.035 \AA$; N(imidazole) $-\mathrm{H}, 1.053 \AA$ ). This does not imply that the maximum electron density is located at the proton position, as the maximum is shifted by the dipolar density function directed into the bond.

(2) The hydrogen atom asphericity is usually described by a dipole charge density function oriented along the $\mathrm{X}-\mathrm{H}$ axes. However, for an accurate description of the hydrogen atom polarization in the molecule or crystal, and thus for an accurate evaluation of electrostatic interaction energy, the addition of a cylindrically symmetric quadrupole density function is required. The density quadrupole moment is related to the product of two p-type polarization functions of the quantum mechanical basis set of the hydrogen atom. ${ }^{1}$ Unfortunately, due to the strong correlation of these moments with the atomic thermal vibrations, the straightforward refinement of the hydrogen atom quadrupole moments from experimental X-ray diffraction is not feasible. As this problem does not exist for the multipole refinements of the static theoretical structure factors, the following approach was adopted.

A reasonable linear relation (linear correlation coefficient $R$ equal to 0.77 ) between the ratio of dipole to quadrupole

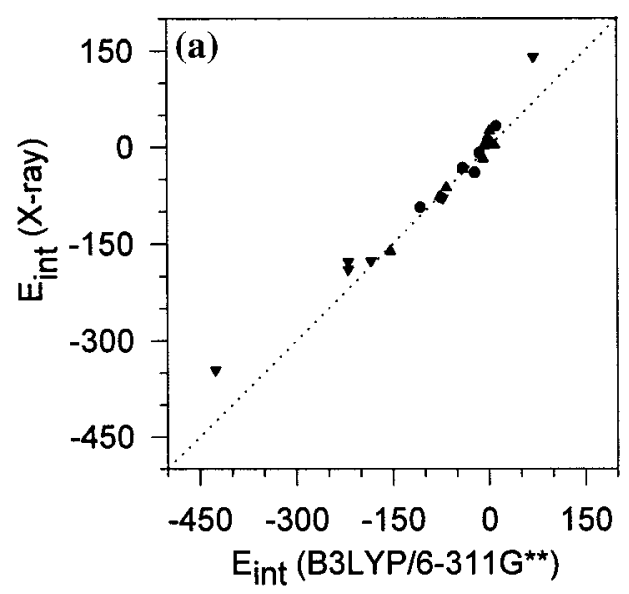

populations $\left(P_{10} / P_{20}\right)$ of the hydrogen atoms participating in $\mathrm{H}$-bonds and the hydrogen-to-acceptor distance is obtained from the refinement of the theoretical structure factors (Figure 3). The ratio decreases with shortening of the hydrogen bond length, reflecting decreased polarization of the hydrogen atom density into the covalent bond as the H-bond strength increases. This linear dependence was introduced as a constraint in the multipole refinement of the charge density of the H-bond hydrogen atoms. Monopolar and dipolar terms only were refined for the $\mathrm{H}$ atoms not participating in strong $\mathrm{H}$-bonds.

Intermolecular Interactions. The ECDA results are compared with those from the supermolecule DFT calculations in Figure 4. The agreement is quite satisfactory, taking into account that the supermolecule calculations do not reflect many-body interactions in the crystal, and better than in earlier work on DL-histidine. ${ }^{14}$ The agreement is best for interaction energies in the -200 to $20 \mathrm{~kJ} / \mathrm{mol}$ range (Figure $4 \mathrm{~b}$ ) but noticeably worse for the two very strong interactions in the glycylglycine crystal (Figure 4a). We note that the theoretical and experimental values of $E_{\text {int }}$, the intermolecular interaction energy in the dimer, should not be quantitatively associated with the hydrogen bond energy, as the intermolecular hydrogen bonds are not the only, and not necessarily the main contributions to the intermolecular interaction energy.

Not unexpectedly, the strongest intermolecular interactions in the dipeptide and the $\alpha$-amino acids takes place between amino and carboxylate groups. In the crystals under consideration the $\mathrm{N}-\mathrm{H} \cdots \mathrm{O}$ hydrogen bonds account for the energetically most favorable centrosymmetric dimer configuration. In this configuration, two identical interactions link the carboxylate and amino groups of the molecules related by inversion centers at $1-X, 1-Y, 1-Z$ in glycylglycine and $-X, 1-Y,-Z$ in DL-histidine ${ }^{14}$ and DL-proline (Figure 2). The hydrogen bonding interaction in such centric dimers is reinforced by the intermolecular dipole-dipole interaction. As the GlyGly molecule possesses the largest dipole moment of the species treated, its dimer displays the highest intermolecular interaction energy (Figure 4 ) and the shortest hydrogen bonds, the $\mathrm{H} \cdots \mathrm{O}$ distances being $1.83,1.93$, and $2.10 \AA$ in the GlyGly, His, and Pro ${ }^{\circ} \mathrm{H}_{2} \mathrm{O}$ dimers, respectively.

A second comparison of theory and experiment is based on the theoretical calculations on the crystal. To make this comparison, the theoretical distributed multipole moments were obtained by multipole refinement of the static theoretical structure factors. Results are shown in Figure 5a, while the electrostatic contributions are illustrated separately in Figure

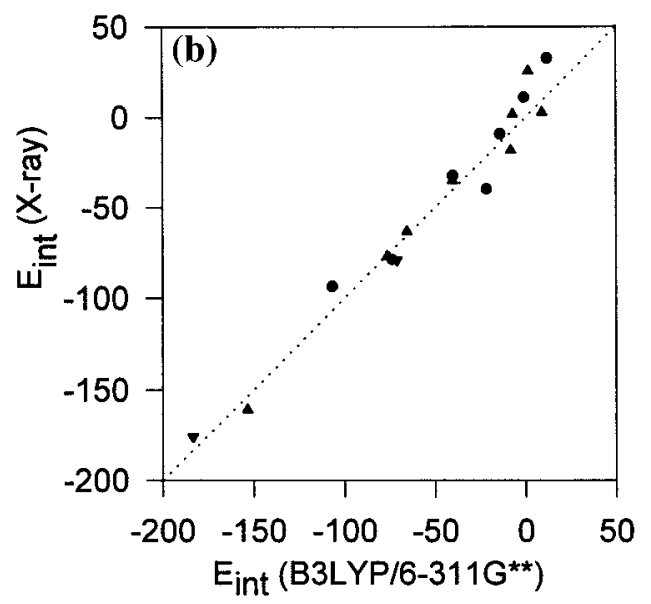

Figure 4. Experimental (ECDA) versus theoretical (supermolecule) intermolecular interaction energies [kJ/mol] in the dimers of DL-proline ( $)$, DL-histidine $(\mathbf{\Lambda})$, and glycylglycine $(\boldsymbol{\nabla})$. Figure $4 \mathrm{~b}$ is an enlargement of the central area of Figure $4 \mathrm{a}$. The dotted line represents exact agreement. 


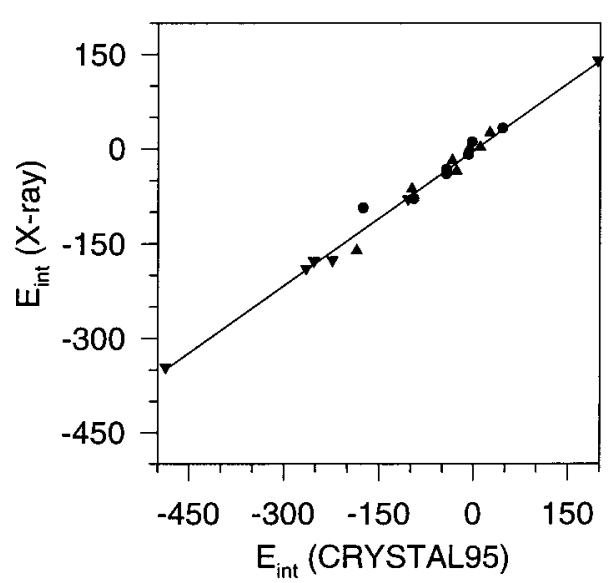

(a)

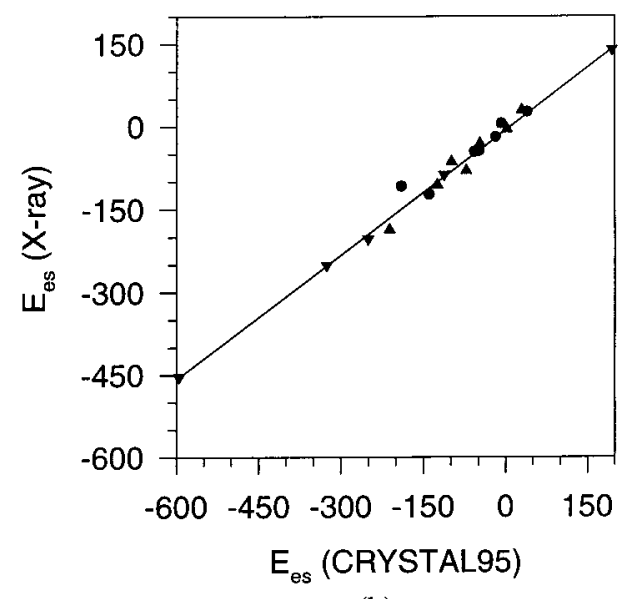

(b)

Figure 5. Comparison of experimental (ECDA) and theoretical (based on periodic HF calculations) intermolecular interaction energies: (a) total and (b) electrostatic contributions only [kJ/mol]. Symbols as in Figure 4. The linear fits $(R=0.99)$ correspond to the equations: (a) $E_{\text {int }}(\mathrm{X}$-ray) $=$ $-0.96(322)+0.72(2) E_{\text {int }}(\mathrm{PHF})$ and $(\mathrm{b}) E_{\mathrm{es}}(\mathrm{X}-\mathrm{ray})=-2.78(369)+0.76(2) E_{\mathrm{es}}(\mathrm{PHF})$

TABLE 2: The Difference between the Energy of the Molecule in the Crystal and the Energy of the Isolated Molecule with the Crystal Geometry Term (Eq 6) (kJ/mol)

\begin{tabular}{lccc}
\hline \multicolumn{1}{c}{ crystal } & $\begin{array}{c}\text { experimental CD } \\
\text { approach }\end{array}$ & $\begin{array}{c}\text { CRYSTAL95 } \\
\text { HF/6-21G** }\end{array}$ & $\begin{array}{c}\text { CRYSTAL95 } \\
\text { HF/6-31G** }\end{array}$ \\
\hline DL-histidine & -240.6 & -244.7 & -234.0 \\
DL-proline. $\mathrm{H}_{2} \mathrm{O}$ & -211.0 & -216.2 & \\
glycylglycine & -353.0 & -362.4 & -357.6
\end{tabular}

5b. The linear fit $\left(E_{\mathrm{es}}(\mathrm{X}\right.$-ray $)=2.8(37)+0.76(2) E_{\mathrm{es}}(\mathrm{PHF}), R$ $=0.99)$ between the two sets of electrostatic energies is quite remarkable. However, the deviation of the slope from $45^{\circ}$ implies a systematic enhancement of the interaction energies from the PHF-derived electron densities. This is in accordance with the well-known overestimation of the molecular dipole moments by the HF calculation, evident in Table 1, which is generally attributed to the neglect of electron correlation. The results suggest that electrostatic energies based on charges from HF calculations should be scaled by a factor of $0.76(2)$. This is within three standard deviations of the value of 0.81 , used in calculations of intermolecular interactions by Price and coworkers, ${ }^{32}$ thus providing quantitative support for this procedure.

Lattice Energies. While the correlation between experiment and theory discussed above is quite reasonable, individual interactions may show noticeable displacement from the straight lines. At least in part the deviations may due to the absence of many-body interactions in the dimer calculations and the limitations of the PHF approach. The lattice energies represent an integrated measure of the interactions and form an additional basis for comparison.

The $E 1$ term (difference between the crystal energy and the energy of the molecules with the crystal geometry, eq 6) of the lattice energy was evaluated by summation of the pairwise intermolecular interactions over an increasing number of neighboring unit cells until convergence was reached within \pm 1 $\mathrm{kJ} / \mathrm{mol}$. Because of the molecular electroneutrality and the zero dipole moment of the unit cell, convergence was achieved rapidly. Summations over 125,125 , and 343 unit cells were needed in the calculations for the DL-histidine, DL-proline, and glycylglycine crystals, respectively.

The ECDA and PHF results for the E1 energy term are presented in Table 2. The differences between the PHF/6-21G** and $\mathrm{PHF} / 6-31 \mathrm{G}^{* *}$ are rather small, indicating that a reasonable estimate of the BSSE has been obtained through the CP correction. The agreement between theory and experiment is
TABLE 3: The Difference between the Energy of the Molecule with the Crystal Geometry and the Isolated Molecule with Optimized Geometry (Eq 8) $(\mathrm{kJ} / \mathrm{mol})$

\begin{tabular}{lc}
\hline molecule & GAUSSIAN94 HF/6-311G** \\
\hline histidine & 109.8 \\
proline $\cdot \mathrm{H}_{2} \mathrm{O}$ & 50.2 \\
glycylglycine & 78.1
\end{tabular}

TABLE 4: Lattice Energies ( $\mathrm{kJ} / \mathrm{mol})$

\begin{tabular}{lccc}
\hline \multicolumn{1}{c}{ crystal } & $\begin{array}{c}\text { experimental CD } \\
\text { approach }\end{array}$ & $\begin{array}{c}\text { CRYSTAL95 } \\
\text { HF/6-21G** }\end{array}$ & $\begin{array}{c}\text { CRYSTAL95 } \\
\text { HF/6-31G** }\end{array}$ \\
\hline DL-histidine & -131.2 & -134.9 & -124.2 \\
DL-proline & -172.5 & -177.7 & \\
glycylglycine & -274.9 & -284.3 & -279.5
\end{tabular}

within $10 \mathrm{~kJ} / \mathrm{mol}$ in all three cases. However, agreement at this level may be due to fortuitous cancellation of errors inherent in the approximations made. For example, the error introduced by the neglect of the dispersion energy in the PHF calculations is at least partially canceled by the enhancement of the electrostatic interactions in the HF method.

To calculate $E 2$ (eq 7), geometry optimizations of the isolated His, Pro, and GlyGly molecules were performed. In all three cases, intramolecular hydrogen bonds were formed (Figure 1, right), as indicated by the formation of topological bond paths between the interacting atoms. ${ }^{17}$ The most striking geometry change occurs in the His molecule. The imidazole ring of this molecule rotates by about $180^{\circ}$ to allow intramolecular hydrogen bonding between the lone pair of the $\mathrm{N} 2$ and the $\mathrm{H} 4$ hydrogen atom. We note that the isolated molecule configuration is close to that found in the L-histidine crystal. ${ }^{33}$ As a result of the noticeable relaxation of the molecular geometries, the $E 2$ term is over $100 \mathrm{~kJ} / \mathrm{mol}$ for DL-histidine and about $80 \mathrm{~kJ} / \mathrm{mol}$ for glycylglycine (Table 3 ). The dissociation energy of the Pro• $\mathrm{H}_{2} \mathrm{O}$ entity is found to be $11.7 \mathrm{~kJ} / \mathrm{mol}$.

The lattice energies are obtained as the sum of $E 1$ and $E 2$ (eq 5). The agreement between the theory and experiment is again quite satisfactory (Table 4), as it must be, given the agreement for $E 1$ and the identical $E 2$ contribution applied to both theory and experiment. Comparison with calorimetric lattice energies would provide an independent test for both theory and experiment. While experimental sublimation enthalpies are apparently not yet available for the crystals under consideration, the $\Delta H_{\text {sub }}$ at $442 \mathrm{~K}$ for L-histidine has been reported as $142 \pm 8 \mathrm{~kJ} / \mathrm{mol}^{3}{ }^{34}$ Since the difference between sublimation enthalpies of racemates and enantiomorphs tends 
to be small, ${ }^{35}$ the experimental value for L-histidine provides a reasonable basis for comparison with the DL-histidine results. After applying a correction estimated at $7 \mathrm{~kJ} / \mathrm{mol}^{36}$ to reduce the $\Delta H_{\text {sub }}(442 \mathrm{~K})$ to the zero temperature value, the calorimetric result exceeds the ECDA and PHF (HF/6-31G**) values by $12 \%$ and $17 \%$, respectively. An underestimation of the lattice energy by the periodic HF calculations was found previously for the urea $(20 \%),{ }^{25}$ formamide $(28 \%),{ }^{26}$ and ice VIII $(21 \%)^{24}$ crystals. For the $\mathrm{HCN}$ crystal the lattice energy was overestimated by $15 \% .^{23}$

\section{Future Prospects}

The calibration of experiment and theory, presented above, provides a basis for use of experimental charge densities in the evaluation of interactions in and between larger molecules, which are less easily treated with advanced theoretical methods. Side chain disorder and solvent interactions are to be treated in future application to the structure of peptides and small proteins. The presence of a macroscopic dipole moment must be taken into account in the evaluation of lattice energies of polar crystals. Such effects will be included in further studies on more complex systems.

Acknowledgment. We thank Dr. T. Koritsanszky for providing the low-temperature data on DL-proline, Dr. M. D. Towler for valuable advice on the use of the CRYSTAL95 program, and Dr. J. S. Chickos for discussions on experimental lattice energies. Support of this work by the National Science Foundation (CHE9615586), the National Institute of Health (GM56829), and the U.S. Department of Energy (DE-FG0286ER45231) is gratefully acknowledged. The Center for Computational Research at SUNY/Buffalo is supported by Grant DBI9871132 from the National Science Foundation.

\section{References and Notes}

(1) Coppens, P. X-ray Charge Densities and Chemical Bonding; Oxford University Press: New York, 1997.

(2) Koritsanszky, T.; Flaig, R.; Zobel, D.; Krane, H.-G.; Morgenroth, W.; Luger, P. Science 1998, 279, 356-358.

(3) Flaig, R.; Koritsanszky, T.; Janczak, J.; Krane, H.-G.; Morgenroth, W.; Luger, P. Angew. Chem., Int. Ed. Engl. 1999, 38, 1397-1400.

(4) Koritsanszky, T.; Howard, S.; Richter, T.; Su, Z.; Mallinson, P. R.; Hansen, N. K. XD-A Computer Program Package for Multipole Refinement and Analysis of Electron Densities from Diffraction Data. User Manual; Free University: Berlin, 1995.

(5) Spackman, M. A. J. Chem. Phys. 1986, 85, 6587-6601.

(6) Kim, Y. S.; Gordon, R. G. J. Chem. Phys. 1974, 66, 1842-1850.

(7) Spackman, M. A. J. Chem. Phys. 1986, 85, 6579-6586.

(8) Mitchell, J. B. O.; Price, S. L. J. Comp. Chem. 1990, 11, 12171233. Coombes, D. S.; Price, S. L.; Willock, D. J.; Leslie, M. J. Phys. Chem. 1996, 100, 7352-7360.

(9) Buckingham, A. D. In Intermolecular Interactions: from Diatomics to Biopolymers; Pullman, B., Ed.; Wiley and Sons: Chichester, New York, 1978; pp 1-67.

(10) According to the Spackman's definition the penetration term is the energy of the interaction of each deformation density fragment with spherical atomic density terms on the other monomer. This energy is evaluated approximately within central multipole expansion scheme. The overlap correction to the interaction energy between deformation fragments on two monomers (i.e., overlap correction to the $\left.E_{\mathrm{es}}^{\mathrm{mul}}(l>0)\right)$ is neglected in his model.

(11) Stone, A. J. The Theory of Intermolecular Forces; International Series of Monographs on Chemistry 32; Oxford University Press: Oxford, 1996.

(12) Kaplan, I. G. Theory of Molecular Interactions; Studies in Physical and Theoretical Chemistry; Elsevier: Amsterdam, 1986; Vol. 42.

(13) Spackman, M. A. J. Phys. Chem. 1987, 91, 3179-3186. Spackman, M. A.; Weber, H. P.; Craven, B. M. J. Am. Chem. Soc. 1988, 110, 775782 .

(14) Coppens, P.; Abramov, Yu. A.; Carducci, M.; Korjov, B.; Novozhilova, I.; Alhambra, C.; Pressprich, C. J. Am. Chem. Soc. 1999, 121, $2585-2593$.

(15) Abramov, Yu. A.; Volkov, A. V.; Coppens, P. Chem. Phys. Lett. 1999, $311,81-86$.

(16) Wu, G.; Coppens, P. To be published.

(17) Bader, R. F. W. Atoms in Molecules-A Quantum Theory; Clarendon Press: Oxford, 1990.

(18) Coombes, D. S.; Price, S. L. Presented at BCA Charge Density Meeting, Durham, UK, December 1995.

(19) Dovesi, R.; Saunders: V. R.; Roetti, C.; Causà, M.; Harrison, N. M.; Orlando, R.; Aprà, E. CRYSTAL95 User's Manual; University of Torino: Torino, Italy, 1996.

(20) Binkley, J. S.; Pople, J. A.; Hehre, W. J. J. Am. Chem. Soc. 1980, 102, 939-946.

(21) Hariharan, P. C.; Pople, J. A. Theor. Chim. Acta 1973, 28, 213222.

(22) Dovesi, R.; Causà, M.; Orlando, R.; Roetti, C.; Saunders: V. R. J. Chem. Phys. 1990, 12, 7402-7411.

(23) Platts, J. A.; Howard, S. T. J. Chem. Phys. 1996, 11, 4668-4674.

(24) Ojame, L.; Hermansson, K.; Dovesi, R.; Roetti, C.; Saunders, V. R. J. Chem. Phys. 1994, 100, 2128-2138.

(25) Gatti, C.; Saunders, V. R.; Roetti, C. J. Chem. Phys. 1994, 101, $10686-10696$

(26) Howard, S. T.; Huke, J. P.; Mallinson, P. R.; Gatti, C. Unpublished results.

(27) Towler, M. D. Private communications.

(28) Monkhorst, H. J.; Pack, J. D. Phys. Rev. B 1976, 13, 5188-5192.

(29) Gilat, G. J. J. Comput. Phys. 1972, 10, 432-465.

(30) Boys, S. F.; Bernardi, F. Mol. Phys. 1970, 19, 553-566.

(31) Frisch, M. J.; Trucks, G. W.; Schlegel, H. B.; Gill, P. M.; Johnson, B. G.; Robb, M. A.; Cheeseman, J. R.; Keith, T.; Petersson, G. A.; Montgomery J. A.; Raghavachari, K.; Al-Laham, M. A.; Zakrzewski, V. G.; Ortiz, J. V.; Foresman, J. B.; Cioslowski, J.; Stefanov, B. B. Nanayakkara, A.; Challacombe, M.; Peng, C. Y.; Ayala, P. Y.; Chen, W.; Wong, M. W.; Andres, J. L.; Replogle, E. S.; Gomperts, R.; Martin, R. L.; Fox, D. J.; Binkley, J. S.; Defrees, D. J.; Baker, J.; Stewart, J. P.; HeadGordon, M.; Gonzalez, C.; Pople, J. A. Gaussian 94, revision E.2; Gaussian Inc.: Pittsburgh, PA, 1995.

(32) Price, S. L.; Andrews, J. S.; Murray, C. W.; Amos, R. D. J. Am. Chem. Soc. 1992, 114, 8268-8276.

(33) Averbuch-Pouchet, M. T. Z. Kristallogr. 1993, 207, 111-120. Wu, G.; Abramov, Yu.; Coppens, P. To be published.

(34) Gaffney, J. S.; Pierce, R. C.; Friedman, L. J. Am. Chem. Soc. 1977 99, 4293.

(35) Chickos, J. S.; Hesse, D. G. Struct. Chem. 1991, 2, 33-40. Larsen, S.; Marthi, K. Acta Crystallogr. B 1999, 53, 803-811. Chickos, J. S. Private communications.

(36) Chickos, J. S. In Molecular Structure and Energetics, Physical Measurements; Liebman, J. F., Greenberg, A., Eds.; VCH Publishers: New York, 1987; Vol. 2, pp 67-150. 